\title{
Analysis of paper pressing: the saturated one-dimensional case
}

\section{Bežanović* C.J. van Duijn, and E.F. Kaasschieter}

Department of Mathematics and Computer Science, Eindhoven University of Technology, PO Box 513, 5600 MB Eindhoven, The Netherlands

Key words Paper pressing, nonlinear diffusion, interior boundary, cross-conditions. MSC (2000) 35B05, 35B50, 35B65, 35R05, 76S05

We derive a one-dimensional model that describes pressing of water saturated paper in the press-section of the paper machine. The model involves two nonlinear diffusion equations which are coupled across an internal boundary. Existence and uniqueness as a number of qualitative properties are demonstrated. Further, computational results for some concrete cases are discussed.

\section{Introduction and motivation}

In paper production, water is removed from the paper web (paper pulp or simply paper) in two sections. The first one is a press-section, in which water is squeezed out of the paper web into the felt by applying a pressure pulse as the paper together with the felt passes through a press nip (see Figures 1 and 2). Excessive water is removed in the dryer-section by an expensive and energy consuming process. Even a small improvement in the efficiency of the press-section may lead to a reduction of the drying time and consequently to a saving of the energy consumption. This motivates a better understanding of the presssection. That is why much research, both experimental and theoretical, has been carried out to understand the ongoing process. This research led to the development of extended nip presses, new felts with higher permeabilities, multi-layered felts and the application of a heat flux $[17,20]$.

Still, challenging questions remain to thoroughly understand the pressing process. Additional knowledge needs to be acquired to quantify, for example, the influence of the air, the mechanical behaviour of the paper web and the magnitude and the direction of flow. Experimental approach is limited by the high processing speed of the paper $\left(\sim 10 \mathrm{~m} \mathrm{~s}^{-1}\right)$ and therefore the small time-scale $\left(\sim 10^{-2} \mathrm{~s}\right)$, and by the small length-scale of the paper thickness $\left(\sim 10^{-4} \mathrm{~m}\right)$. This motivates mathematical modelling of the pressing process, see for instance [10, 13, 15, 16, 19, 21].

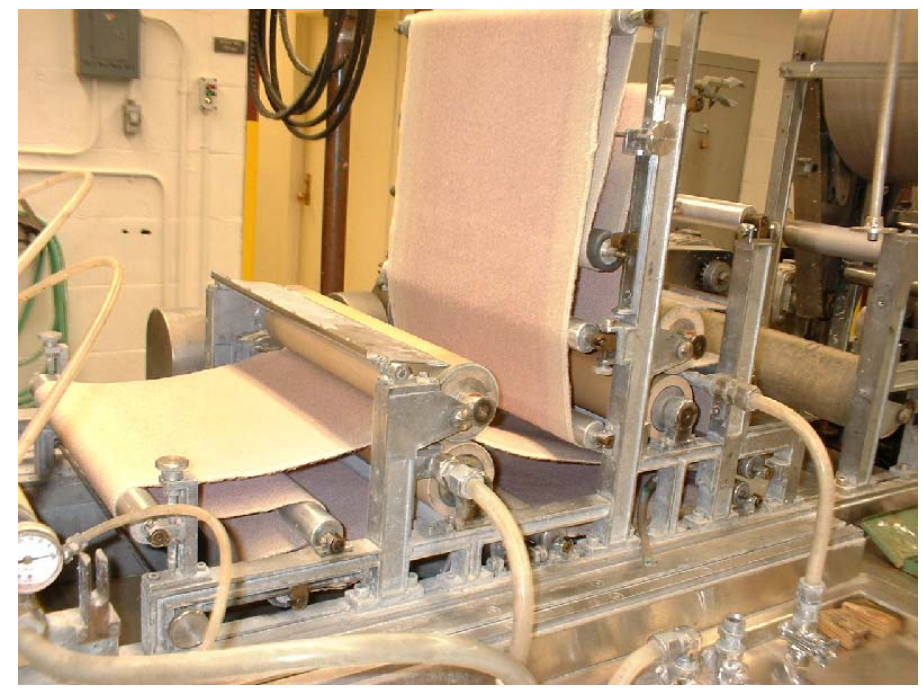

Fig. 1 Press-section of paper machine.

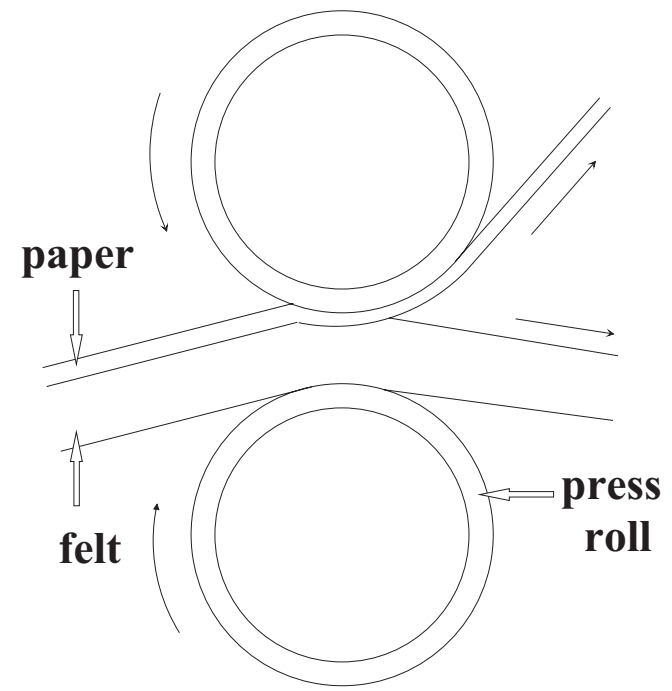

Fig. 2 Details of press-section.

However much of these papers involve numerical studies. In this paper we present a rigorous mathematical analysis, addressing existence, uniqueness and qualitative properties of solutions of a simplified model. Though limited in the practical context,

\footnotetext{
* Corresponding author, e-mail d.bezanovic@tue.nl
} 
our proposed model is a first step towards a more throughout understanding of the processes in the press-section. Moreover, the questions posed are non-trivial from the mathematical point of view. By recognizing the analogy with two-phase flow in heterogenous porous media, much use could be made from ideas and techniques developed in Bertsch et al [2].

This paper is outlined as follows. In Section 2 we sketch the physical background. The mathematical formulation, involving the nonlinear diffusion equations in the paper and felt as well as the matching conditions across the contact interface, is presented in Section 3. In Section 4 we prove existence by a suitable regularization technique and in Section 5 we prove a comparison result with uniqueness as a consequence. Some qualitative properties are discussed in Section 6 and we conclude, in Section 7, with two numerical examples.

\section{Physical model}

\subsection{Assumptions}

We consider paper and felt as a system having two deformable porous layers. These layers are assumed to be completely saturated. This assumption is justified, at least for paper, since it is observed that most of the air is removed from the paper in the early part of the nip. Because the layers are mixtures of a solid and a fluid phase, we use the continuum theory of mixtures to describe this process. The flow of water through the layers is governed by Darcy's law, which is an approximation of the momentum balance for the water phase.

To obtain a simple, one-dimensional model, only transversal fluid flow is considered. This is motivated by a scaling argument which uses the fact that the longitudinal flow is at least one order of magnitude smaller than the transversal flow, see for instance [20]. We also assume that the mechanical behaviour of the layers is perfectly elastic. The permeability of the medium changes with the deformation and the water and the solid phase are incompressible.

\subsection{Governing equations}

In this section we present the fundamental balance laws for each layer. Since layers experience deformation we use a Lagrangian approach. We denote by $z$ the spatial transversal coordinate (perpendicular to the machine direction) and by $Z$ the transversal material coordinate. The relation between them is given by the mapping $Z \mapsto z(Z, t)$ for all $t \in\left(0, t_{f i n}\right)$, such that $z(Z, 0)=Z$, for all $Z$. Here $t_{\text {fin }}$ is the time needed for a paper particle to move through the press-nip.

Since the model is one-dimensional, volumes are described by intervals. Corresponding to an arbitrary reference volume $V_{0}=\left(Z_{0}^{1}, Z_{0}^{2}\right)$ in the material coordinate, there exist a time dependent volume $V_{t}=\left(z_{t}^{1}, z_{t}^{2}\right)$ in the spatial coordinate. Here $\left(z_{0}^{1}, z_{0}^{2}\right)=\left(Z_{0}^{1}, Z_{0}^{2}\right)$. We further assume that paper occupies the interval $0<Z<h_{0 p}$ and felt $h_{0 p}<Z<h_{0 p}+h_{0 f}$, where $h_{0 p}$ and $h_{0 f}$ are the initial thicknesses of paper and felt.

The solid mass balance equation is given by

$$
\frac{d}{d t} \int_{V_{t}}(1-\phi) d z=0
$$

where $\phi$ is the porosity. Since water moves with respect to the solid, the water mass balance reads

$$
\frac{d}{d t} \int_{V_{t}} \phi d z+q\left(y_{t}, t\right)-q\left(z_{t}, t\right)=0 .
$$

Here $q$ is the relative specific discharge, given by $q=\phi\left(v_{w}-v_{s}\right)$, where $v_{w}$ and $v_{s}$ are the velocities of water and solid, respectively. The flow is governed by Darcy's law

$$
q=-\frac{k}{\mu} \frac{\partial p_{w}}{\partial z}
$$

where $p_{w}$ and $\mu$ are, respectively, the pressure and the viscosity of the water and $k$ the permeability of the medium. From the balance of total momentum it follows that, see [10,3],

$$
\frac{\partial p_{T}}{\partial z}=0
$$

Here $p_{T}$ is the total applied pressure. It is distributed over the phase pressures according to

$$
p_{T}=p_{s}+p_{w}
$$

where $p_{s}$ is the structural pressure. It is the part of the total pressure responsible for the deformation of the solid matrix. 


\subsection{Constitutive equations}

We assume that the layers deform only in the transversal $z$-direction and that visco-elastic effects can be disregarded $[21,15]$. This results in a structural pressure which is a given function of the strain $\epsilon$ :

$$
p_{s}=P_{s}(\epsilon)
$$

where

$$
\epsilon=\frac{\partial U}{\partial Z}
$$

and where $U$ is the displacement

$$
U(Z, t)=z(Z, t)-z(Z, 0)=z(Z, t)-Z .
$$

In a state of compression we have $-1<\epsilon^{*}<\epsilon<0$, where $\epsilon^{*}$ is the strain corresponding to maximal deformation: $\phi=0$, i.e. no void space.

Referring to paper and felt, we denote their properties by the additional subscripts $p$ and $f$. Throughout this paper we assume

$$
\begin{array}{ll}
\left(A_{P}\right): \quad & P_{s i}:\left(\epsilon_{i}^{*}, 0\right] \mapsto[0, \infty) \text { is a smooth function such that } \\
& P_{s i}\left(\epsilon_{i}^{*+}\right)=+\infty, P_{s i}(0)=0 \text { and } P_{s i}^{\prime}(\epsilon)<0 \text { for } \epsilon_{i}^{*}<\epsilon<0, i=p, f .
\end{array}
$$

The permeability $k$ changes due to the deformation of the medium. This is modelled by assuming $k=k(\phi)$ satisfying

$$
\begin{array}{ll}
\left(A_{k}\right): & k_{i}:[0,1] \mapsto[0, \infty) \text { is a smooth function such that } \\
& k_{i}(0)=0 \text { and } k_{i}^{\prime}(\phi)>0 \text { for } 0<\phi<1, i=p, f .
\end{array}
$$

\subsection{Initial, boundary and cross conditions}

Since we are considering a two layer (paper-felt) system, we need in addition to initial and boundary conditions, also cross conditions at the interface between the layers. In particular we consider the following situation:

- The initial (reference) configuration is undeformed and for each layer the initial state is uniform.

- The bottom surface of the paper is in contact with a nonpermeable press-roll. This implies the no flow condition $q=0$, see Figure 3. The upper surface of the felt is in contact with a perfectly perforated roll; i.e. there is no flow resistance, implying $p_{w}=0$. Furthermore, $p_{T}$ is given as a function of time.

- Paper and felt are in perfect contact. This means that the discharge $q$ and pressures $p_{s}$ and $p_{w}$ are continuous across the interface.

$$
Z=h_{0 p}+h_{0 f} \text { free flow }
$$

\begin{tabular}{cl} 
FELT & $Z=h_{0 p}$ \\
PAPER & $Z=0$ \\
\hline
\end{tabular}

\section{no flow}

Fig. 3 Two layer structure.

\section{Mathematical model}

\subsection{Derivation of the mathematical model}

We formulate the model in terms of the Lagrangian coordinate $Z$. In the notation below we introduce the superscript $\sim$ to denote dependence on $Z$ : for instance $\phi(z, t)=\phi(z(Z, t), t)=\widetilde{\phi}(Z, t)$. 
For any reference volume $V_{0}$ in either the paper or the felt we have the identity

$$
\int_{V_{t}}(1-\phi) d z=\int_{V_{0}}(1-\widetilde{\phi}) \frac{d z}{d Z} d Z=\int_{V_{0}}(1-\widetilde{\phi})(1+\widetilde{\epsilon}) d Z .
$$

Assuming sufficient smoothness we obtain from (2.1)

$$
\int_{V_{0}} \frac{\partial}{\partial t}((1-\tilde{\phi})(1+\widetilde{\epsilon})) d Z=0
$$

and thus

$$
\frac{\partial}{\partial t}((1-\widetilde{\phi})(1+\widetilde{\epsilon}))=0 \text { for } Z \in\left(0, h_{0 p}\right) \cup\left(h_{0 p}, h_{0 p}+h_{0 f}\right) .
$$

Since the initial configuration is assumed undeformed, the initial strain is zero. Therefore

$$
1+\widetilde{\epsilon}=\frac{1-\widetilde{\phi}_{0}}{1-\widetilde{\phi}} \text { in }\left(0, h_{0 p}\right) \cup\left(h_{0 p}, h_{0 p}+h_{0 f}\right),
$$

where $\phi_{0}$ is the constant initial porosity. Assuming that $q$ in (2.2) is smooth we have

$$
\frac{d}{d t} \int_{V_{t}} \phi d z+\int_{V_{t}} \frac{\partial q}{\partial z} d z=0
$$

Transforming again to the material coordinate $Z$, we obtain

$$
\frac{\partial}{\partial t}(\widetilde{\phi}(1+\widetilde{\epsilon}))+\frac{\partial \widetilde{q}}{\partial Z}=0
$$

and, with Darcy's law (2.3),

$$
\frac{\partial}{\partial t}(\widetilde{\phi}(1+\widetilde{\epsilon}))-\frac{\partial}{\partial Z}\left(\frac{k(\widetilde{\phi})}{\mu(1+\widetilde{\epsilon})} \frac{\partial \widetilde{p}_{w}}{\partial Z}\right)=0 \text { in }\left(0, h_{0 p}\right) \cup\left(h_{0 p}, h_{0 p}+h_{0 f}\right) .
$$

It is convenient to rewrite equation (3.2) in terms of the scaled void ratio

$$
\widetilde{u}:=\widetilde{\phi}(1+\widetilde{\epsilon})>\widetilde{\phi}\left(1+\epsilon^{*}\right) \geq 0 .
$$

Combining this expression with (3.1) gives

$$
\widetilde{\epsilon}=\widetilde{u}-\phi_{0} \text { and } \widetilde{\phi}=\widetilde{\phi}\left(\widetilde{u}, \phi_{0}\right):=\frac{\widetilde{u}}{1+\widetilde{u}-\phi_{0}} .
$$

For the two materials, $\phi_{0}$ and the functions $k$ and $P_{s}$ may be different. Let, for $i=p, f$,

$$
\begin{aligned}
& P_{i}(\widetilde{u}):=P_{s i}\left(\widetilde{u}-\phi_{0 i}\right)=P_{s i}(\widetilde{\epsilon}) \\
& C_{i}(\widetilde{u}):=\frac{k_{i}\left(\widetilde{\phi}\left(\widetilde{u}, \phi_{0 i}\right)\right) \widetilde{\phi}\left(\widetilde{u}, \phi_{0 i}\right)}{\mu \widetilde{u}}=\frac{k_{i}(\widetilde{\phi})}{\mu(1+\widetilde{\epsilon})} .
\end{aligned}
$$

Using (2.4) and (2.5) to replace the water pressure by the structural pressure in (3.2), and applying definitions (3.3), (3.5) and (3.6), gives the equation

$$
\frac{\partial \widetilde{u}}{\partial t}+\frac{\partial}{\partial Z}\left(C_{i}(\widetilde{u}) \frac{\partial P_{i}(\widetilde{u})}{\partial Z}\right)=0, i=p, f .
$$

For $i=p$, equation (3.7) is valid in the domain

$$
W^{p}=\left\{(Z, t): Z \in\left(0, h_{0 p}\right), t \in\left(0, t_{\text {fin }}\right)\right\},
$$

and for $i=f$ in the domain

$$
W^{f}=\left\{(Z, t): Z \in\left(h_{0 p}, h_{0 p}+h_{0 f}\right), t \in\left(0, t_{\text {fin }}\right)\right\} .
$$

Remark 3.1 Relations (3.3) and (3.4) imply

1. $\widetilde{\phi}=0$ (state of complete compression) $\Rightarrow \widetilde{u}=0, \widetilde{\epsilon}=\epsilon^{*}=-\phi_{0}$;

2. $\widetilde{\phi}=\phi_{0}$ (initial state) $\Rightarrow \widetilde{u}=\phi_{0}, \widetilde{\epsilon}=0$. 


\subsection{Initial, boundary and cross conditions}

In terms of the variable $\widetilde{u}$ we have:

\section{Initial condition:}

$$
\widetilde{u}(Z, 0)=\left\{\begin{array}{l}
u_{0 p}=\phi_{0 p} \text { for } Z \in\left(0, h_{0 p}\right), \\
u_{0 f}=\phi_{0 f} \text { for } Z \in\left(h_{0 p}, h_{0 p}+h_{0 f}\right) .
\end{array}\right.
$$

\section{Boundary conditions:}

$Z=0$ : Combining (2.3), (2.5), (2.4) and (3.5) and using the strict monotonicity as assumed in $\left(A_{P}\right)$, the no-flow condition along the bottom implies

$$
\frac{\partial \widetilde{u}}{\partial Z}(0, t)=0 \text { for } t \in\left(0, t_{\text {fin }}\right)
$$

$Z=h_{0 p}+h_{0 f}:$ We assume that the total pressure $p_{T}$ is given by the function $t \mapsto P_{T}(t)$, where $P_{T}$ satisfies:

$$
\begin{array}{ll}
\left(A_{P_{T}}\right): & P_{T}:\left[0, t_{f i n}\right] \rightarrow[0, \infty) \text { is a smooth function such that } \\
& P_{T}(0)=P_{T}(1)=0 \text { and } P_{T}(t)>0 \text { for } 0<t<t_{f i n} .
\end{array}
$$

Combining (2.5) and (3.5), the pressure condition $p_{w}=0$ implies the boundary condition

$$
\widetilde{u}\left(h_{0 p}+h_{0 f}, t\right)=u_{b}(t):=P_{f}^{-1}\left(P_{T}(t)\right) \text { for } t \in\left(0, t_{f i n}\right)
$$

\section{Cross conditions:}

1. Continuity of flow requires

$$
\left(C_{p}(\widetilde{u}) \frac{\partial \widetilde{P}_{p}}{\partial Z}\right)\left(h_{0 p}^{-}, t\right)=\left(C_{f}(\widetilde{u}) \frac{\partial \widetilde{P}_{f}}{\partial Z}\right)\left(h_{0 p}^{+}, t\right) \text { for } t \in\left(0, t_{f i n}\right)
$$

where $C_{i}(i=p, f)$ is given by (3.6).

2. Continuity of pressure implies

$$
P_{p}\left(\widetilde{u}\left(h_{0 p}^{-}, t\right)\right)=P_{f}\left(\widetilde{u}\left(h_{0 p}^{+}, t\right)\right) \text { for } t \in\left(0, t_{f i n}\right)
$$

Remark 3.2 Since $P_{T}(t)>0$ for all $t \in\left(0, t_{\text {fin }}\right)$, the boundary condition $u_{b}$ satisfies $0<u_{b}(t)<u_{0 f}$ for all $t \in\left(0, t_{\text {fin }}\right)$. This means that we consider the case of compression, with $\epsilon\left(h_{0 p}+h_{0 f}, t\right)<0$ for all $0<t<t_{\text {fin }}$.

Remark 3.3 Without loss of generality we assume throughout this paper that $u_{0 f}<u_{0 p}$.

\section{Existence of solution}

\subsection{Problem formulation}

We scale the equation by redefining

$$
t:=\frac{t}{t_{\text {fin }}}
$$

and by introducing

$$
x:=\frac{Z}{h_{0 p}+h_{0 f}}, x_{c}:=\frac{h_{0 p}}{h_{0 p}+h_{0 f}} \text { and } P_{i}:=\frac{P_{i}}{P_{f}^{*}} \text { for } i=p, f,
$$

where $P_{f}^{*}$ is a characteristic structural pressure of the felt.

Under this scaling $W^{p} \mapsto Q^{p}=\left(0, x_{c}\right) \times(0,1)$ and $W^{f} \mapsto Q^{f}=\left(x_{c}, 1\right) \times(0,1)$. Further we set $Q^{*}:=Q^{p} \cup Q^{f}$, $Q=(0,1) \times(0,1)$ and we drop the 'tildes' in the notation. Then the problem to be considered is

$$
u_{t}+f_{x}=0 \text { in } Q^{*},
$$


where

$$
f=D p_{x}
$$

with

$$
\begin{aligned}
& p=P(u, x)=P_{p}(u)+H_{x_{c}}(x)\left(P_{f}(u)-P_{p}(u)\right), \\
& D=D(u, x)=D_{p}(u)+H_{x_{c}}(x)\left(D_{f}(u)-D_{p}(u)\right),
\end{aligned}
$$

and

$$
D_{i}(u):=\frac{t_{f i n} P_{f}^{*}}{\left(h_{0 p}+h_{0 f}\right)^{2}} C_{i}(u), i=p, f .
$$

Here $f$ denotes the water flux and $H_{x_{c}}$ the shifted Heaviside function:

$$
H_{x_{c}}(x)=\left\{\begin{array}{l}
0 \text { for } x<x_{c} \\
1 \text { for } x>x_{c}
\end{array}\right.
$$

The subscripts $t$ and $x$ in (4.1) denote partial differentiation with respect to these variables. Note that (4.3) and (4.4) involve only dimensionless nonlinearities.

Assumptions $A_{p}$ and $A_{k}$ motivate the following properties for the pressures $P_{i}$ and the diffusivities $D_{i}$ :

$$
\begin{array}{ll}
\left(H_{P}\right): & P_{i}:(0, \infty) \mapsto \mathbb{R} \text { with } P_{i} \in C^{3+\alpha}((0, \infty)) \text { for some } \alpha \in(0,1), \\
& P_{i}\left(0^{+}\right)=+\infty, P_{i}\left(u_{0 i}\right)=0 \text { and } P_{i}^{\prime}(u)<0 \text { for all } u>0, i=p, f . \\
\left(H_{D}\right): & D_{i}:[0, \infty) \mapsto[0, \infty) \text { with } D_{i} \in C^{2+\alpha}([0, \infty)) \text { for some } \alpha \in(0,1), \\
& D_{i}(0)=0 \text { and } D_{i}(u)>0, \text { for all } u>0, i=p, f .
\end{array}
$$

Note that we have extended the domain of definition of the nonlinearities $P_{i}$ and $D_{i}$ to the half line $[0, \infty)$. This is done for technical reasons. Later we show that $u$ satisfies the natural bounds $0<u<u_{0 i}$ in $Q^{i}(i=p, f)$.

The initial condition is written as

$$
u(x, 0)=u_{0}(x):=u_{0 p}+H_{x_{c}}(x)\left(u_{0 f}-u_{0 p}\right) \text { for } 0<x<1
$$

and the boundary conditions are

$$
u_{x}(0, t)=0 \text { and } u(1, t)=u_{b}(t) \text { for all } 0 \leq t \leq 1
$$

With respect to $u_{b}$ we assume:

$$
\begin{aligned}
\left(H_{u_{b}}\right): \quad & u_{b}:[0,1] \mapsto\left(0, u_{0 f}\right] \text { with } u_{b} \in C^{2+\alpha / 2}([0,1]), \text { for some } \alpha \in(0,1), \\
& u_{b}(0)=u_{0}(1)=u_{0 f}, u_{b}^{\prime}(0)=0, u_{b}(t)<u_{0 f} \text { for } 0<t<1 \text { and } \\
& u_{b}^{\prime} \text { has a finite number of sign changes in }[0,1] .
\end{aligned}
$$

Remark 4.1 In the context of paper-pressing the function $u_{b}$ has only one extremum (maximum) in [0,1]. Therefore the limitation on the number of sign changes poses no restriction.

Remark 4.2 To carry out the steps and manipulations in the proofs of this paper, we need certain smoothness for the approximate solutions introduced in Subsection 4.2. As will be explained, this is guaranteed by the smoothness assumptions and compatibility conditions in the above hypotheses. In fact, in the approximate problems we need $u_{b}^{\prime \prime}(0)=0$. This is achieved by a modification of $u_{b}$ which disappears when passing to the limit.

The matching conditions at $x=x_{c}$ are

$$
p\left(x_{c}^{-}, t\right)=p\left(x_{c}^{+}, t\right) \text { and } f\left(x_{c}^{-}, t\right)=f\left(x_{c}^{+}, t\right) \text { for all } 0 \leq t \leq 1
$$

Thus the model combines two nonlinear diffusion equations with matching conditions across the interface $x=x_{c}$. The latter is the particularity of the model. To study existence, uniqueness and some qualitative properties we borrow ideas from Bertsch et al [2]. We start with the formulation:

Problem $\mathcal{P}$ : Find $u: Q^{*} \rightarrow(0, \infty)$ and $p, f: Q \rightarrow \mathbb{R}$ such that 
(i) $u \in C^{2,1}\left(Q^{i}\right) \cap C\left(\overline{Q^{i}}\right)$ and $u(1, \cdot)=u_{b}(\cdot)$ in $[0,1]$;

(ii) $p \in C(\bar{Q})$ and $p=P_{i}(u)$ in $Q^{i}$;

(iii) $f \in L^{\infty}((0,1) ; B V(0,1)), f=D_{i}(u) P_{i}^{\prime}(u) u_{x}$ in $Q^{i}$ and

$$
\iint_{Q} u \zeta_{t} d x d t+\iint_{Q} f \zeta_{x} d x d t+\int_{0}^{1} u_{0}(x) \zeta(x, 0) d x=0
$$

for all $\zeta \in H^{1}(Q) \cap C(\bar{Q})$ vanishing at $x=1$ and at $t=1$. Here $u_{0}$ is given by (4.7).

The formulation implies directly

$$
\begin{aligned}
& u_{t}+\left(D_{i}(u) P_{i}^{\prime}(u) u_{x}\right)_{x}=0 \text { in } Q^{i}, \\
& f\left(x_{c}^{-}, \cdot\right)=f\left(x_{c}^{+}, \cdot\right) \text { a.e. in }[0,1],
\end{aligned}
$$

and, as stated, continuity of the pressure across the peper-felt interface.

\subsection{Regularization}

We construct a solution of Problem $\mathcal{P}$ as the limit of a sequence of solutions of corresponding regularized problems. Regularized functions are denoted by the upper index $n$. Throughout this section we consider $n \in \mathbb{N}$ and $n$ sufficiently large. Let

$$
H_{x_{c}}^{n}(x)=\psi\left(n\left(x-x_{c}\right)\right), x \in[0,1],
$$

where $\psi: \mathbb{R} \rightarrow[0,1]$ is a $C^{\infty}$-function satisfying

$$
\psi(s)=\left\{\begin{array}{l}
0 \text { for } s \leq-1 \\
\text { strictly increasing for }-1<s<1 \\
1 \text { for } s \geq 1
\end{array}\right.
$$

Further, let

$$
P^{n}(u, x)=P_{p}(u)+H_{x_{c}}^{n}(x)\left(P_{f}(u)-P_{p}(u)\right),
$$

and

$$
D^{n}(u, x)=D_{p}(u)+H_{x_{c}}^{n}(x)\left(D_{f}(u)-D_{p}(u)\right) .
$$

The initial value $u_{0}$ is regularized such that

$$
P^{n}\left(u_{0}^{n}(x), x\right)=0 \text { for all } x \in[0,1] .
$$

The functions $u_{0}^{n}$ satisfy

\section{Proposition 4.1}

(i) $u_{0}^{n} \in C^{\infty}([0,1])$;

(ii) $u_{0}^{n}=\left\{\begin{array}{l}u_{0 p} \text { for } x<x_{c}-1 / n, \\ u_{0 f} \text { for } x>x_{c}+1 / n ;\end{array}\right.$

(iii) $u_{0}^{n \prime}(x) \leq 0$ for $0 \leq x \leq 1$.

Proof. The first property follows from direct differentiation of (4.12). The strict monotonicity of $P_{i}$ and the properties of $H_{x_{c}}^{n}$ result in (ii). To show (iii) we first note that $P^{n}$ is a convex combination of $P_{p}$ and $P_{f}$. Since both these functions are positive for $u<u_{0 f}$ and negative for $u>u_{0 p}$, (4.12) implies $u_{0 f} \leq u_{0}^{n} \leq u_{0 p}$. Differentiating (4.12) with respect to $x$ and using $P_{p}(u) \geq P_{f}(u)$ for $u_{0 f} \leq u \leq u_{0 p}$, we have

$$
u_{0}^{n \prime}=-\frac{P_{x}^{n}}{P_{u}^{n}}=-H_{x_{c}}^{n \prime}(x) \frac{P_{f}(u)-P_{p}(u)}{P_{u}^{n}} \leq 0 .
$$


The regularized problems to be studied are:

$$
\mathcal{P}^{n}:\left\{\begin{array}{l}
u_{t}+\left(D^{n}(u, x)\left(P^{n}(u, x)\right)_{x}\right)_{x}=0 \text { in } Q \\
u(1, t)=u_{b}(t) \text { for } 0 \leq t \leq 1 \\
u_{x}(0, t)=0 \text { for } 0 \leq t \leq 1 \\
u(x, 0)=u_{0}^{n}(x) \text { for } 0 \leq x \leq 0
\end{array}\right.
$$

Since $D_{i}(0)=0$ and $P_{i}\left(0^{+}\right)=\infty$ (with no rates specified) we want to avoid points in $Q$ where $u$ vanishes. At $t=0$ we have

$$
0<u_{0 f} \leq u_{0}^{n} \text { in }[0,1]
$$

Therefore, Problem $\mathcal{P}^{n}$ is non-degenerate at $t=0$ and consequently a unique local (for $0<t<\delta_{n}$ ) classical solution $u^{n}$ exists, see for instance [14, Theorem 4.1, p. 558], giving

$$
u^{n} \in C^{2+\beta, 1+\beta / 2}\left(\bar{Q}_{\delta_{n}}\right) \text { for any } \beta \in(0,1),
$$

where $Q_{\delta_{n}}=(0,1) \times\left(0, \delta_{n}\right)$.

This solution can be continued in $Q$ as long as it remains bounded and bounded away from zero.

Let $Q_{n}=(0,1) \times\left(0, t_{n}\right)$ denote the maximal existence domain for $u^{n}$. Below we show that $t_{n}=1$ by constructing a uniform upper and (positive) lower bound for $u^{n}$. For this purpose we use the pressure formulation. Setting $p^{n}=P^{n}\left(u^{n}, x\right)$ and differentiating (4.10) we obtain

$$
p_{t}^{n}=P_{u}^{n} u_{t}^{n}=-P_{u}^{n}\left(D^{n} p_{x}^{n}\right)_{x} \text { in } Q_{n}
$$

Along the lateral boundaries we have for $0<t<t_{n}$

$$
p_{x}^{n}(0, t)=P_{p}^{\prime}\left(u^{n}\right) u_{x}^{n}(0, t)=0
$$

and

$$
p^{n}(1, t)=P_{f}\left(u_{b}(t)\right)=P_{T}(t)
$$

At $t=0$ we have by (4.12),

$$
p^{n}(\cdot, 0)=0 \text { in }[0,1]
$$

With $\bar{p}:=\max _{0 \leq t \leq 1} P_{T}(t)$ the maximum principle for equation (4.15) gives

$$
0<p^{n}<\bar{p} \text { in } Q_{n}
$$

Using again (4.10) we obtain the uniform bounds

$$
0<\underline{u}<u^{n}<u_{0 p} \text { in } Q_{n}
$$

The positive lower bound is given by $\underline{u}:=P_{\min }^{-1}(\bar{p})$, where $P_{\min }(u)=\min \left\{P_{f}(u), P_{p}(u)\right\}$ (see Figure 4).

The uniform bounds imply that $t_{n}=1$ for each $n \in \mathbb{N}$. Thus we have obtained

Lemma 4.1 Problem $\mathcal{P}^{n}$ has a unique classical solution $u^{n} \in C^{2+\beta, 1+\beta / 2}(\bar{Q})$ satisfying

$$
\underline{u} \leq u^{n} \leq u_{0 p} \text { in } \bar{Q}
$$

for all $n \in \mathbb{N}$, n sufficiently large.

Remark 4.3 Differentiating the $u^{n}$-equation with respect to $t$ gives a linear parabolic equation for $u_{t}^{n}$ in $Q$. The smoothness assumptions in the hypotheses combined with (4.14) imply Hölder continuity for the coefficients in this equation. Together with $H_{u_{b}}$ and Remark 4.2, in particular $u_{b}^{\prime \prime}(0)=0$, we find from [14, Theorem 5.2, p. 320]

$$
u_{t}^{n} \in C^{2+\beta, 1+\beta / 2}\left(\bar{Q}_{\delta_{n}}\right) .
$$

We need this smoothness in the following section when considering the flux equation. 


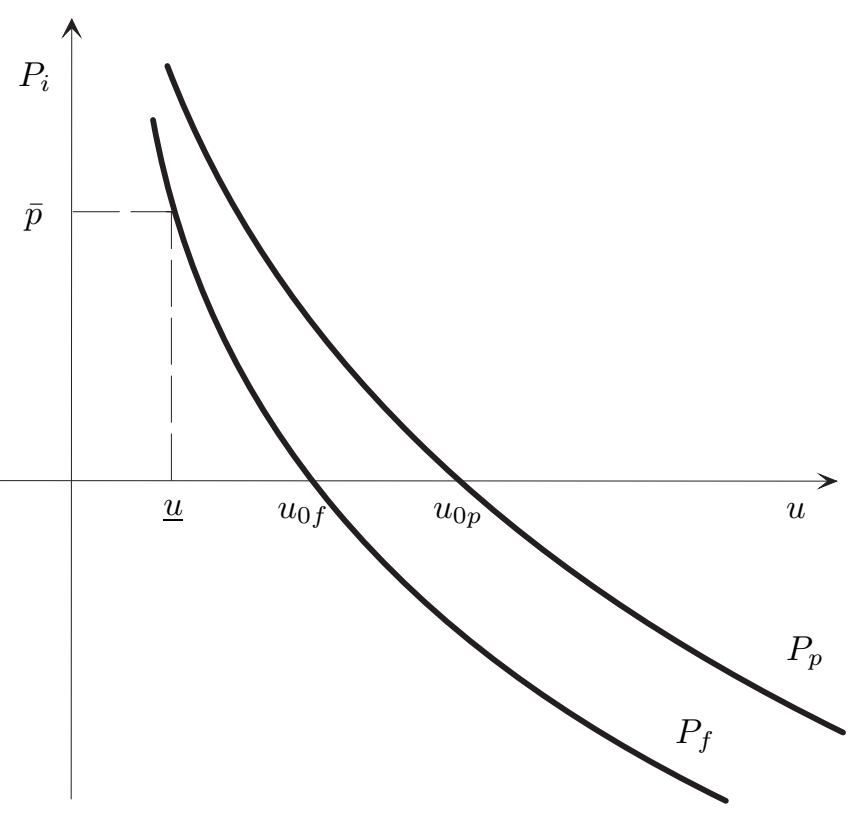

Fig. 4 Pressure curves for paper and felt. If the curves are ordered as sketched, we have $\underline{u}=P_{f}^{-1}(\bar{p})$.

\subsection{Flux estimates}

In this section we give some uniform estimates for the regularized flux

$$
f^{n}:=D^{n} p_{x}^{n}
$$

We start with the following boundary estimate.

Lemma 4.2 There exists $L>0$ such that

$$
\left|f^{n}(1, t)\right| \leq L, \text { for all } 0 \leq t \leq 1
$$

for all $n \in \mathbb{N}$, $n$ sufficiently large.

Pr o of. To simplify the notation we drop the superscript $n$. We use a barrier function argument in the set

$$
Q^{\delta}=(1-\delta, 1) \times(0,1) \subset Q^{f},
$$

where $0<\delta<1-\left(x_{c}+1 / n\right)$. This choices implies that $u$ satisfy

$$
u_{t}+\left(D_{f}(u)\left(P_{f}(u)\right)_{x}\right)_{x}=0 \text { in } Q^{\delta}
$$

We transform (4.20) by setting

$$
v=\phi(u)=-\int_{u_{0 f}}^{u} D_{f}(s) P_{f}^{\prime}(s) d s .
$$

Note that $\phi^{\prime}(u)>0$ for $\underline{u} \leq u \leq u_{0 p}$. Hence $\beta(v):=\phi^{-1}(v)$ exists and is strictly increasing such that $0<\beta_{*} \leq \beta^{\prime}(v) \leq \beta^{*}$ for all $\phi(\underline{u})=: \underline{v} \leq v \leq v_{0 p}:=\phi\left(u_{0 p}\right)$. Further we note that $f=-v_{x}$ in $Q^{\delta}$. To prove the lemma we therefore construct a boundary estimate on $v_{x}$.

The transformed function $v$ satisfies

$$
(\beta(v))_{t}=v_{x x} \text { in } Q^{\delta}
$$

with

$$
\left.\begin{array}{l}
v(1, t)=\phi\left(u_{b}(t)\right)=: v_{b}(t), \\
\underline{v} \leq v(1-\delta, t) \leq v_{0 p}
\end{array}\right\} \text { for } 0 \leq t \leq 1
$$


and

$$
v(x, 0)=0 \text { for } 1-\delta \leq x \leq 1 .
$$

Bellow we show that for appropriately chosen $C_{1}$ and $C_{2}$, the function

$$
\widetilde{v}(x, t)=v_{b}(t)+C_{1}(1-x)-C_{2}(1-x)^{2}
$$

is a supersolution in $Q^{\delta}$. Consider the linear operator

$$
\mathcal{L}(\alpha)=\beta^{\prime}(v) \alpha_{t}-\alpha_{x x} .
$$

Then $\mathcal{L}(v)=0$ and

$$
\mathcal{L}(\widetilde{v})=\beta^{\prime}(v) v_{b}^{\prime}+2 C_{2}>0 \text { in } Q^{\delta}
$$

for $C_{2}$ sufficiently large. Further

$$
\widetilde{v}(1-\delta, t)=v_{b}(t)+C_{1} \delta-C_{2} \delta^{2}>v_{0 p}
$$

provided $C_{1}$ large enough, and

$$
\widetilde{v}(1, t)=v_{b}(t)
$$

for all $0 \leq t \leq 1$. Finally

$$
\widetilde{v}(x, 0)=C_{1}(1-x)-C_{2}(1-x)^{2} \geq 0,
$$

again for $C_{1}$ large enough. By the maximum principle we have $v \leq \widetilde{v}$ in $Q^{\delta}$. Since $v(1, t)=\widetilde{v}(1, t)$ we obtain

$$
v_{x}(1, t) \geq \widetilde{v}_{x}(1, t)=-C_{1} \text { for } 0 \leq t \leq 1 .
$$

An upper bound is constructed accordingly.

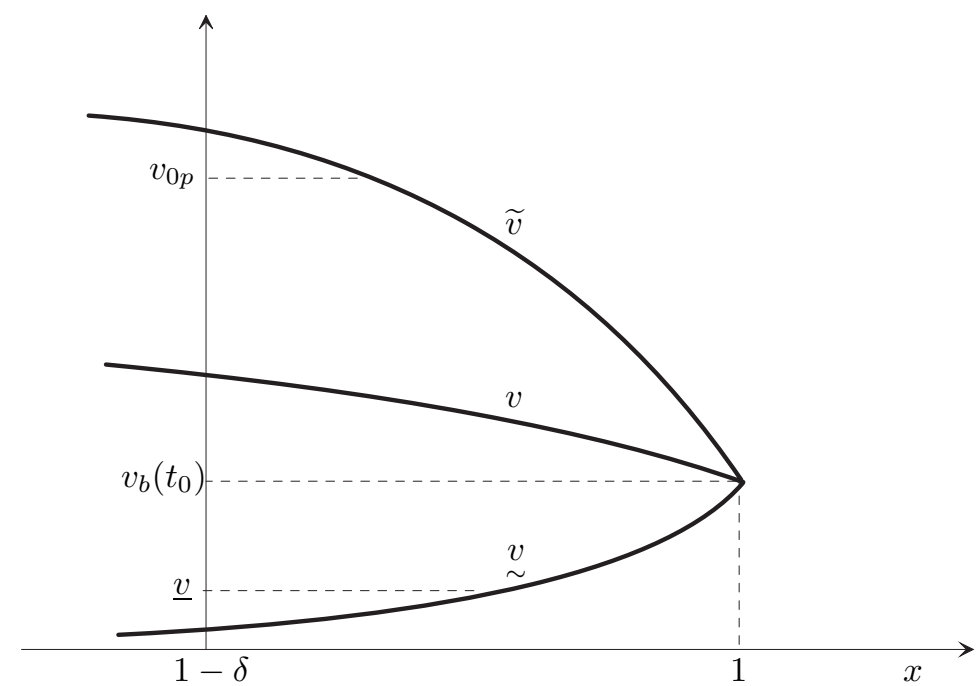

Fig. 5 Subsolution $\underset{\sim}{v}$ ) and supersolution $(\widetilde{v})$ of $v$ in $Q^{\delta}$, at $t=t_{0} \in(0,1)$.

Lemma 4.3 The sequence $\left\{f^{n}\right\}$ is uniformly bounded in $L^{\infty}(Q)$.

Proof. Again we drop superscript $n$ from the notation. We want to derive an equation for the flux. Since $u_{t}=-f_{x} \in$ $C^{2+\alpha, 1+\alpha / 2}(\bar{Q})$, we are allowed to differentiate (4.19) with respect to $t$. This gives

$$
f_{t}=a f_{x x}+b f_{x} \text { in } Q,
$$


where $a=-D P_{u}$ and $b=-\left(D_{u} P_{u} u_{x}+D P_{u u} u_{x}+D_{u} P_{x}+D P_{x u}\right)$. Lemma 4.2 and expressions (4.16) and (4.12) imply

$$
\left.\begin{array}{l}
\mid \begin{array}{l}
f(1, t) \mid<L, \\
f(0, t)=0
\end{array}
\end{array}\right\} \text { for } 0 \leq t \leq 1
$$

and

$$
f(x, 0)=0 \text { for } 0 \leq x \leq 1 \text {. }
$$

Then the maximum principle yields $|f|<L$ in $Q$.

The next lemma gives the global and uniform $x$-regularity of the fluxes $f^{n}$ (see also Bertsch et al [2]).

Lemma 4.4 The sequence $\left\{f^{n}\right\}$ is uniformly bounded in $L^{\infty}\left((0,1) ; W^{1,1}(0,1)\right)$.

Pro of. Here too we drop the superscript $n$ from the notation. Using equation (4.21), we evaluate for fixed $n \in \mathbb{N}, \epsilon>0$ and $0<t<1$

$$
\begin{aligned}
\frac{d}{d t} \int_{0}^{1} \sqrt{\left(f_{x}\right)^{2}+\epsilon} & =\int_{0}^{1} \frac{f_{x} f_{x t}}{\left(\left(f_{x}\right)^{2}+\epsilon\right)^{1 / 2}}=\left.\frac{f_{x} f_{t}}{\left(\left(f_{x}\right)^{2}+\epsilon\right)^{1 / 2}}\right|_{x=0} ^{1}-\int_{0}^{1} \frac{\epsilon f_{x x}}{\left(\left(f_{x}\right)^{2}+\epsilon\right)^{3 / 2}} f_{t} \\
& =\frac{f_{x} f_{t}}{\left(\left(f_{x}\right)^{2}+\epsilon\right)^{1 / 2}}(1, t)-\int_{0}^{1} \frac{\epsilon a\left(f_{x x}\right)^{2}}{\left(\left(f_{x}\right)^{2}+\epsilon\right)^{3 / 2}}-\int_{0}^{1} \frac{\epsilon b f_{x}}{\left(\left(f_{x}\right)^{2}+\epsilon\right)^{3 / 2}} f_{x x} \\
& \leq \frac{f_{x} f_{t}}{\left(\left(f_{x}\right)^{2}+\epsilon\right)^{1 / 2}}(1, t)-\int_{0}^{1} \frac{\epsilon f_{x}}{\left(\left(f_{x}\right)^{2}+\epsilon\right)^{3 / 2}} b f_{x x} .
\end{aligned}
$$

Integrating this inequality in $(0, t)$ gives

$$
\int_{0}^{1} \sqrt{f_{x}^{2}(t)+\epsilon}-\sqrt{\epsilon} \leq \int_{0}^{t} \frac{f_{x} f_{t}}{\left(\left(f_{x}\right)^{2}+\epsilon\right)^{1 / 2}}(1, s) d s-\int_{0}^{t} \int_{0}^{1} \frac{\epsilon f_{x}}{\left(\left(f_{x}\right)^{2}+\epsilon\right)^{3 / 2}} b f_{x x} .
$$

The second term on the right can be written as

$$
\int_{0}^{t} \int_{0}^{1} g_{\epsilon}\left(f_{x}\right) b f_{x x}
$$

where

$$
g_{\epsilon}(s)=\frac{\epsilon s}{\left(s^{2}+\epsilon\right)^{3 / 2}}, s \in \mathbb{R}
$$

This function is uniformly bounded and satisfies $\lim _{\epsilon \downarrow 0} g_{\epsilon}(s)=0$ pointwisely in $\mathbb{R}$. Using this and the fact that $b f_{x x} \in$ $L^{1}((0,1) \times(0, t))$ we obtain from Lebesgue's dominated convergence theorem

$$
\lim _{\epsilon \downarrow 0} \int_{0}^{t} \int_{0}^{1} \frac{\epsilon f_{x}}{\left(\left(f_{x}\right)^{2}+\epsilon\right)^{3 / 2}} b f_{x x}=0
$$

for each $t \in(0,1)$ and $n \in \mathbb{N}$.

To estimate the boundary term we note that

$$
f_{x}(1, t)=-u_{t}(1, t)=-u_{b}^{\prime}(t)
$$

Thus,

$$
\frac{f_{x} f_{t}}{\left(\left(f_{x}\right)^{2}+\epsilon\right)^{1 / 2}}(1, t)=-\frac{u_{b}^{\prime}(t)}{\left(\left(u_{b}^{\prime}(t)\right)^{2}+\epsilon\right)^{1 / 2}} f_{t}(1, t) \rightarrow\left\{\begin{array}{l}
0 \text { in }\left\{u_{b}^{\prime}=0\right\}, \\
-f_{t} \text { in }\left\{u_{b}^{\prime}>0\right\}, \\
f_{t} \text { in }\left\{u_{b}^{\prime}<0\right\},
\end{array} \text { as } \epsilon \downarrow 0 .\right.
$$

Letting $\epsilon \rightarrow 0$ in (4.22) and using (4.23) and (4.24) gives the estimate

$$
\int_{0}^{1}\left|f_{x}(t)\right| \leq 2(N+1) L, \text { for all } t \in(0,1) \text { and } n \in \mathbb{N} \text {. }
$$

Here $N$ is the number of sign changes of $u_{b}^{\prime}$ in $(0,1)$ and $L$ is the constant from Lemma 4.2. 


\subsection{Existence}

We first show that the sequence $\left\{u^{n}\right\}$ is equicontinuous away from $x_{c}$. Bellow we use the notation, for $\mu>0$,

$$
\begin{aligned}
& N(\mu)=\min \left\{n \in \mathbb{N}, n>\frac{1}{\mu}\right\}, \\
& Q(\mu)=\left\{(x, t): x \in\left[0, x_{c}-\mu\right) \cup\left(x_{c}+\mu, 1\right], t \in[0,1]\right\} .
\end{aligned}
$$

Then we have:

Lemma 4.5 The sequence $\left\{u^{n}\right\}_{n \geq N(\epsilon)}$ is equicontinuous in $Q(\epsilon)$ for each sufficiently small $\epsilon>0$.

Pro of. Fix $0<\epsilon<\min \left\{x_{c}, 1-x_{c}\right\}$. The uniform bounds in Lemma 4.1 and Lemma 4.3, and the particular choice of regularisations (4.10) and (4.11) imply that solutions $u^{n}$, with $n \geq N(\epsilon)$, are uniformly Lipschitz continuous with respect to $x$ in $Q(1 / N(\epsilon))$. The smoothness and boundedness of the coefficients of the $u^{n}$-equation allow us to apply [8]. As a result we obtain that for $n \geq N(\epsilon)$ the solutions $u^{n}$ are uniformly Hölder continuous (exponent $1 / 2$ ) with respect to $t$ in $Q(\epsilon) \subset Q(1 / N(\epsilon))$. This proves the assertion.

We are now in a position to apply Ascoli's Theorem [11] and the (usual) diagonal procedure to obtain a subsequence of $\left\{u^{n}\right\}$, again denoted by $\left\{u^{n}\right\}$, and a function $u \in L^{\infty}(Q) \cap C\left(Q^{*}\right)$ such that

$$
\begin{aligned}
& u^{n} \rightarrow u \text { pointwisely in } Q^{*}, \\
& u^{n} \rightarrow u \text { uniformly in } \overline{Q(\epsilon)} \text { for any } \epsilon>0,
\end{aligned}
$$

as $n \rightarrow \infty$. Clearly

$$
\underline{u} \leq u \leq u_{0 p} \text { in } Q^{*}
$$

and $u(1, \cdot)=u_{b}(\cdot)$ in $[0,1]$.

The corresponding sequence of fluxes $\left\{f^{n}\right\}$ is uniformly bounded in $L^{\infty}(Q) \subset L^{2}(Q)$. Hence there exists a subsequence, here too denoted by $\left\{f^{n}\right\}$, and a function $f \in L^{\infty}(Q)$ such that

$$
f^{n} \rightarrow f \text { weakly in } L^{2}(Q) .
$$

Since

$$
\iint_{Q}\left\{u^{n} \zeta_{t}+f^{n} \zeta_{x}\right\} d x d t+\int_{0}^{1} u_{0}^{n}(x) \zeta(x, 0) d x=0
$$

for all test functions $\zeta$ as in Problem $\mathcal{P}$, we obtain along the subsequence $n \rightarrow \infty$

$$
\iint_{Q}\left\{u \zeta_{t}+f \zeta_{x}\right\} d x d t+\int_{0}^{1} u_{0}(x) \zeta(x, 0) d x=0 .
$$

Recalling

$$
p_{x}^{n}=\frac{f^{n}}{D^{n}}
$$

it follows from Lemma 4.1, Lemma 4.3 and $\left(H_{D}\right)$ that there exists a constant $K>0$ such that

$$
\left|p^{n}\left(x_{c}+\delta, t\right)-p^{n}\left(x_{c}-\delta, t\right)\right| \leq 2 \delta K
$$

for each $t \in[0,1]$ and for all $\delta>0$. Hence, the global pressure

$$
p:=\left\{\begin{array}{l}
P_{p}(u) \text { in } Q^{p} \\
P_{f}(u) \text { in } Q^{f}
\end{array}\right.
$$

satisfies $p \in C(\bar{Q})$. As a consequence $u \in C\left(\overline{Q^{i}}\right)$ for $i=p, f$.

Next we fix $\zeta \in C_{0}^{\infty}\left(Q^{i}\right)$. Then

$$
\begin{aligned}
0 & =\iint_{Q^{i}}\left\{u^{n} \zeta_{t}+D_{i}\left(u^{n}\right) P_{i}^{\prime}\left(u^{n}\right) u_{x}^{n} \zeta_{x}\right\} d x d t \\
& =\iint_{Q(\epsilon)}\left\{u^{n} \zeta_{t}+D_{i}\left(u^{n}\right) P_{i}^{\prime}\left(u^{n}\right) u_{x}^{n} \zeta_{x}\right\} d x d t
\end{aligned}
$$


for $\epsilon$ such that supp $(\zeta) \subset Q(\epsilon)$ and for all $n \geq N(\epsilon)$. By the uniform convergence of $u^{n}$ and the weak convergence of $f^{n}$ we obtain

$$
\iint_{Q^{i}}\left\{u \zeta_{t}+D_{i}(u) P_{i}(u) u_{x} \zeta_{x}\right\} d x d t=0
$$

for all $\zeta \in C_{0}^{\infty}\left(Q^{i}\right)$. Here $u_{x}=f /\left(D_{i}(u) P_{i}^{\prime}(u)\right)$ in $Q^{i}$.

Hence $u$ is a weak solution of

$$
u_{t}+\left(D_{i}(u) P_{i}^{\prime}(u) u_{x}\right)_{x}=0 \text { in } Q^{i}
$$

and $u$ is Hölder continuous away from $\left\{x=x_{c}\right\}$ (as a consequence of the proof of Lemma 4.5). Moreover, away from $\left\{x=x_{c}\right\}$, the sequence $\left\{u^{n}\right\}$ is uniformly bounded in $C^{2+\beta, 1+\beta / 2}$ (see, for instance, [7, Theorem 5, p. 64]). It follows that $u \in C^{2,1}\left(Q^{*}\right)$ and (4.27) is satisfied classically (see also [4]). Finally, inherited from Lemma 4.4, we have $f \in L^{\infty}((0,1), B V(0,1))$. This completes the existence proof:

Theorem 4.1 The triple $(u, p, f)$ is a solution of Problem $\mathcal{P}$.

Restricted to $Q^{i}$, the solution $u$ has the same smoothness as the approximations $u^{n}$. In particular

$$
u_{t} \in C^{2+\alpha, 1+\alpha / 2}\left(\widetilde{Q}^{i}\right)(i=p, f)
$$

where

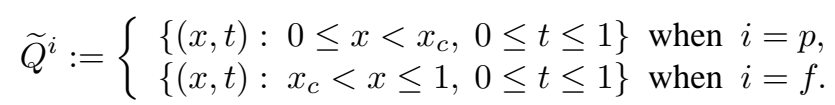

This follows along the same lines, see for instance Remark 4.3. Let

$$
\left.\begin{array}{l}
u_{f}(t):=u\left(x_{c}^{+}, t\right) \\
u_{p}(t):=u\left(x_{c}^{-}, t\right)
\end{array}\right\} \text { for } 0 \leq t \leq 1
$$

Continuity of the pressure $p$ in $\bar{Q}$ implies $u_{p}, u_{f} \in C([0,1])$. This can be improved by using the boundedness of the flux.

Lemma 4.6 $u_{p}, u_{f} \in C^{1 / 4}([0,1])$.

Proof. We only prove the assertion for $u_{p}$. For any fixed $0 \leq t_{1}<t_{2} \leq 1$ we consider the rectangle $R^{p}=\left(0, x_{c}\right) \times$ $\left(t_{1}, t_{2}\right)$. Then

$$
\iint_{R^{p}} u_{t} \phi(x) d x d t+\int_{t_{1}}^{t_{2}} f\left(x_{c}^{-}, t\right) \phi\left(x_{c}\right) d t-\iint_{R^{p}} f \phi^{\prime}(x) d x d t=0,
$$

for any $\phi \in W^{1, \infty}\left(0, x_{c}\right)$. Taking $\phi(x)=u\left(x, t_{2}\right)-u\left(x, t_{1}\right)$ gives

$$
\int_{0}^{x_{c}}\left\{u\left(x, t_{2}\right)-u\left(x, t_{1}\right)\right\}^{2} d x \leq K\left(t_{2}-t_{1}\right),
$$

where $K$ is a positive constant involving the Lipschitz constant of $u$. This inequality implies uniform Hölder continuity of $u$ with respect to $t$ (exponent $1 / 4)$. Details are given in [6].

Remark 4.4 Theorem 4.1 gives existence for the piecewise constant initial condition (4.7). This result extends straightforwardly to

$$
u_{0}(x)=\left\{\begin{array}{l}
u_{0 p}(x) \text { for } 0 \leq x \leq x_{c} \\
u_{0 f}(x) \text { for } x_{c} \leq x \leq 1
\end{array}\right.
$$

for sufficiently smooth, positive functions $u_{0 p}$ and $u_{0 f}$, satisfying the compatibility conditions $u_{0 p}^{\prime}(0)=0, P_{p}\left(u_{0 p}\left(x_{c}\right)\right)=$ $P_{f}\left(u_{0 f}\left(x_{c}\right)\right)$ and $u_{0 f}^{\prime}(1)=u_{0 f}^{\prime \prime}(1)=0$.

We conclude this section by showing that compression occurs in $Q^{f}$ as well.

Lemma 4.7 We have

$$
\underline{u}<u<u_{0 f} \text { in } Q^{f}
$$

and

$$
g(\underline{u})<u<u_{0 p} \text { in } Q^{p} .
$$

Here $g=P_{p}^{-1} \circ P_{f}$ and $\underline{u}$ is the lower bound from Lemma 4.1. 
Proof. Suppose $u>u_{0 f}$ somewhere in $Q^{f}$. Then the maximum principle gives that $u>u_{0 f}$ somewhere on the parabolic boundary of $Q^{f}$. However, $u(1, t)=u_{b}(t) \leq u_{0 f}$ for all $0 \leq t \leq 1$ and $u(x, 0)=u_{0 f}$ for all $x_{c} \leq x \leq 1$. Hence, $u>u_{0 f}$ somewhere on the segment $\left\{(x, t): x=x_{c}, 0 \leq t \leq 1\right\}$. If $\left(x_{c}, t_{c}\right)$ is such a point, then $P_{p}\left(u_{p}\left(t_{c}\right)\right)=P_{f}\left(u_{f}\left(t_{c}\right)\right)<0$, implying $u_{p}\left(t_{c}\right)>u_{0 p}$. This contradicts (4.25). A similar argument gives the lower bound in $Q^{p}$.

\section{Comparison principle}

We start with a comparison result for Problem $\mathcal{P}$ involving arbitrary initial $\left(u_{0}\right)$ and boundary $\left(u_{b}\right)$ data. Throughout we assume that hypotheses $\left(H_{P}\right),\left(H_{D}\right)$ and $\left(H_{u_{b}}\right)$, as well as the conditions from Remark 4.4 are satisfied. The proof uses ideas from [2].

Theorem 5.1 Let $\left(u_{1}, p_{1}, f_{1}\right)$ and $\left(u_{2}, p_{2}, f_{2}\right)$ denote two solutions of Problem $\mathcal{P}$ corresponding to initial/boundary data $\left(u_{0_{1}}, u_{b_{1}}\right)$ and $\left(u_{0_{2}}, u_{b_{2}}\right)$, respectively. Then $u_{0_{1}} \leq u_{0_{2}}$ and $u_{b_{1}} \leq u_{b_{2}}$ in $[0,1]$ imply $u_{1} \leq u_{2}$ in $Q^{*}$.

Proof. We set, for $i=p, f$,

$$
\phi_{i}(u):=-\int_{u_{0 i}}^{u} D_{i}(s) P_{i}^{\prime}(s) d s
$$

Clearly

$$
f= \begin{cases}-\left(\phi_{p}\right)_{x} & \text { in } Q^{p} \\ -\left(\phi_{f}\right)_{x} & \text { in } Q^{f}\end{cases}
$$

Next we test the equation for the difference,

$$
\iint_{Q}\left\{\left(u_{1}-u_{2}\right) \zeta_{t}+\left(f_{1}-f_{2}\right) \zeta_{x}\right\} d x d t+\int_{0}^{1}\left(u_{0_{1}}(x)-u_{0_{2}}(x)\right) \zeta(x, 0) d x=0
$$

with

$$
\zeta=\xi \psi \eta
$$

where

(i) $\xi$ is a $C^{1}$ cut-off function near $x=x_{c}$ :

$$
\xi(x)=\left\{\begin{array}{l}
1 \text { for } 0 \leq x \leq x_{c}-\delta \\
0 \text { for } x_{c}-\delta / 2 \leq x \leq x_{c}+\delta / 2 \\
1 \text { for } x_{c}+\delta \leq x \leq 1
\end{array}\right.
$$

such that $\xi^{\prime}(x) \leq 0$ for $x_{c}-\delta \leq x \leq x_{c}-\delta / 2$ and $\xi^{\prime}(x) \geq 0$ for $x_{c}+\delta / 2 \leq x \leq x_{c}+\delta$.

(ii) $\psi$ is a $C^{1}$ cut-off function near $t=\tau \in(0,1]$ :

$$
\psi(t)=\left\{\begin{array}{l}
1 \text { for } 0 \leq t \leq \tau-\mu \\
0 \text { for } \tau \leq t \leq 1
\end{array}\right.
$$

such that $\psi^{\prime}(t) \leq 0$ for $\tau-\mu<t<\tau$.

(iii) $\eta=S_{\epsilon}\left(\phi_{p}\left(u_{1}\right)-\phi_{p}\left(u_{2}\right)\right)+H_{x_{c}}^{n}(x)\left\{\left(S_{\epsilon}\left(\phi_{f}\left(u_{1}\right)-\phi_{f}\left(u_{2}\right)\right)-S_{\epsilon}\left(\phi_{p}\left(u_{1}\right)-\phi_{p}\left(u_{2}\right)\right)\right\}\right.$,

in which $n$ is taken such that $n>2 / \delta$ and where

$$
S_{\epsilon}(r)=\left\{\begin{array}{l}
0 \text { for } r \leq 0 \\
r / \sqrt{r^{2}+\epsilon^{2}} \text { for } r>0 .
\end{array}\right.
$$

Here, $\delta, \mu$ and $\epsilon$ are sufficiently small positive parameters. Note that for $\epsilon \downarrow 0$,

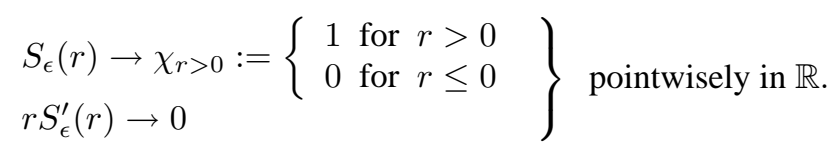


As in Section 4, regularity theory for the equations in the subdomains $Q^{p}$ and $Q^{f}$ gives that both $u_{1}$ and $u_{2}$ satisfy (4.28). Hence we may integrate first term in (5.2) by parts. With $Q_{\tau}=(0,1) \times(0, \tau)$ this gives

$$
\begin{aligned}
\iint_{Q_{\tau}}\left(u_{1}-u_{2}\right)_{t} \xi \psi \eta d x d t & =\iint_{Q_{\tau}}\left(f_{1}-f_{2}\right) \psi \xi^{\prime} \eta d x d t-\sum_{i=p, f} \iint_{Q_{\tau}^{i}}\left(f_{1}-f_{2}\right)^{2} \psi \xi S_{\epsilon}^{\prime}\left(\phi_{i}\left(u_{1}\right)-\phi_{i}\left(u_{2}\right)\right) d x d t \\
& \leq \iint_{Q_{\tau}}\left(f_{1}-f_{2}\right) \psi \xi^{\prime} \eta d x d t .
\end{aligned}
$$

Here $Q_{\tau}^{i}(i=p, f)$ denotes either the paper or the felt part of $Q_{\tau}$.

We fix $\mu, \delta>0$ and let $\epsilon \downarrow 0$. Then (5.4) becomes

$$
\iint_{Q_{\tau}} \xi \psi\left(\left(u_{1}-u_{2}\right)_{+}\right)_{t} d x d t \leq \iint_{Q_{\tau}}\left(f_{1}-f_{2}\right) \psi \xi^{\prime} S_{\epsilon} \chi_{\left\{u_{1}>u_{2}\right\}} d x d t
$$

since $\phi_{i}\left(u_{1}\right) \leq \phi\left(u_{2}\right) \Leftrightarrow u_{1} \leq u_{2}$. Letting $\mu \downarrow 0$ and using the assumption that $u_{0_{1}} \leq u_{0_{2}}$ we obtain

$$
\begin{aligned}
\int_{0}^{1} \xi\left(u_{1}-u_{2}\right)_{+}(\tau) d x & \leq \int_{0}^{\tau}\left\{\int_{x_{c}-\delta}^{x_{c}-\delta / 2}\left(f_{1}-f_{2}\right) \xi^{\prime} \chi_{\left\{u_{1}>u_{2}\right\}} d x+\int_{x_{c}+\delta / 2}^{x_{c}+\delta}\left(f_{1}-f_{2}\right) \xi^{\prime} \chi_{\left\{u_{1}>u_{2}\right\}} d x\right\} d t \\
& =: \int_{0}^{\tau}\left(I_{\delta}^{-}+I_{\delta}^{+}\right) d t .
\end{aligned}
$$

Let $t \in(0, \tau)$ be chosen such that $f^{-}$and $f^{+}$exist. We set $u_{i}^{ \pm}:=u_{i}\left(x_{c}^{ \pm}\right)$, for $i=1,2$, and consider the possibilities:

1. $u_{1}^{+}>u_{2}^{+}$. Then $u_{1}>u_{2}$ in the right neighbourhood of $x=x_{c}$ and $\chi_{\left\{u_{1}>u_{2}\right\}}=1$ in $\left(x_{c}+\delta / 2, x_{c}+\delta\right)$ for $\delta$ sufficiently small. The pressure condition implies $u_{1}^{-}>u_{2}^{-}$and consequently $\chi_{\left\{u_{1}>u_{2}\right\}}=1$ in $\left(x_{c}-\delta, x_{c}-\delta / 2,\right)$, again for small $\delta$. Using this in (5.5) and using

$$
\int_{x_{c}+\delta / 2}^{x_{c}+\delta}\left(f_{1}-f_{2}\right) \xi^{\prime} d x \rightarrow f_{1}^{+}-f_{2}^{+} \text {and } \int_{x_{c}-\delta}^{x_{c}-\delta / 2}\left(f_{1}-f_{2}\right) \xi^{\prime} d x \rightarrow-\left(f_{1}^{-}-f_{2}^{-}\right) \text {as } \delta \rightarrow 0 .
$$

we find

$$
\lim _{\delta \rightarrow 0}\left(I_{\delta}^{-}+I_{\delta}^{+}\right)=\left[f_{1}\right]-\left[f_{2}\right]=0 .
$$

2. $u_{1}^{+}<u_{2}^{+}$. By the same arguments as above $\chi_{\left\{u_{1}>u_{2}\right\}}=0$ in $(-\delta,-\delta / 2) \cup\left(x_{c}+\delta / 2, x_{c}+\delta\right)$, for $\delta$ sufficiently small. Therefore

$$
I_{\delta}^{-}+I_{\delta}^{+}=0
$$

3. $u_{1}^{+}=u_{2}^{+}$. In this case we have to compare the fluxes:

(a) $f_{1}^{+}=f_{2}^{+}$. Then $f_{1}^{-}=f_{2}^{-}$and consequently

$$
\sup _{\left(x_{c}+\delta / 2, x_{c}+\delta\right)}\left(f_{1}-f_{2}\right) \chi_{\left\{u_{1}>u_{2}\right\}} \rightarrow 0 \text { and } \sup _{\left(x_{c}-\delta, x_{c}-\delta / 2\right)}\left(f_{1}-f_{2}\right) \chi_{\left\{u_{1}>u_{2}\right\}} \rightarrow 0 \text { as } \delta \rightarrow 0 .
$$

Thus again

$$
\lim _{\delta \rightarrow 0}\left(I_{\delta}^{-}+I_{\delta}^{+}\right)=0 .
$$

(b) $f_{1}^{+}>f_{2}^{+}$. Then $f_{1}>f_{2}$ and therefore $u_{1 x}<u_{2 x}$ and $u_{1}<u_{2}$ in $\left(x_{c}+\delta / 2, x_{c}+\delta\right)$ for small $\delta$. By flux continuity $f_{1}^{-}>f_{2}^{-}$and thus $u_{1 x}<u_{2 x}$ and $u_{1}>u_{2}$ in $\left(x_{c}-\delta, x_{c}-\delta / 2\right)$ for small $\delta$. Therefore

$$
I_{\delta}^{-}+I_{\delta}^{+}=I_{\delta}^{-} \leq 0 .
$$

(c) $f_{1}^{+}<f_{2}^{+}$. Now $u_{1 x}>u_{2 x}$ and $u_{1}>u_{2}$ in $\left(x_{c}+\delta / 2, x_{c}+\delta\right)$ for $\delta$ small. Like above $f_{1}^{-}<f_{2}^{-}$and therefore $u_{1 x}>u_{2 x}$ and $u_{1}<u_{2}$ in $\left(x_{c}-\delta, x_{c}-\delta / 2\right)$ for small $\delta$. Thus

$$
I_{\delta}^{-}+I_{\delta}^{+}=I_{\delta}^{+} \leq 0 .
$$

Combining these conclusions with (5.5) we get

$$
u_{1}(\cdot, \tau) \leq u_{2}(\cdot, \tau) \text { in }(0,1) \backslash\left\{x_{c}\right\} .
$$

Since $\tau$ was taken arbitrary, the proof is complete.

As a direct consequence we have

Theorem 5.2 Problem $\mathcal{P}$ has at most one solution. 


\section{Some qualitative properties}

The main object of the pressing process is to remove water from paper. Since the phases are assumed incompressible, the amount of water in the paper is measured by its thickness. In unscaled variables the paper thickness is given by

$$
h_{p}(t)=\int_{0}^{h_{p}(t)} d z=\int_{0}^{h_{0 p}}(1+\epsilon(Z, t)) d Z .
$$

Using scaling (3.4) and the scaling of Section 4 we obtain

$$
h_{p}(t):=\frac{h_{p}(t)}{h_{0 p}+h_{0 f}}=\int_{0}^{x_{c}} u(x, t) d x+x_{c}\left(1-\phi_{0 p}\right) .
$$

We first show that the paper thickness decreases during the first time interval where the total applied pressure increases.

Proposition 6.1 Let $t^{*} \in(0,1)$ be such that $P_{T}^{\prime}>0$ in $\left(0, t^{*}\right)$. Then $h_{p}^{\prime}<0$ in $\left(0, t^{*}\right)$.

Proof. We differentiate (6.1) with respect to $t$ and use equation (4.1). This gives

$$
h_{p}^{\prime}(t)=-f\left(x_{c}^{-}, t\right)=-f\left(x_{c}^{+}, t\right)
$$

for $0 \leq t \leq 1$. We use a maximum principle argument for the fluxes to show

$$
f\left(x_{c}, t\right)>0 \text { for } 0<t<t^{*}
$$

which proves the assertion.

From the definition (3.9) it follows that $u_{b}^{\prime} \leq 0$ in $\left[0, t^{*}\right]$. Hence

$$
f_{x}(1, t)=-u_{b}^{\prime}(t) \geq 0 \text { for } 0 \leq t \leq t^{*}
$$

Consequently, the flux cannot attain a minimum along the boundary segment $\left\{(x, t): x=1,0 \leq t \leq t^{*}\right\}$. If the flux would be negative somewhere in $Q_{t^{*}}^{f}=\left(x_{c}, 1\right) \times\left(0, t^{*}\right)$, then it has to reach a negative minimum at some point $\left(x_{c}, t_{0}\right)$, with $0<t_{0} \leq t^{*}$, where

$$
f_{x}\left(x_{c}^{+}, t_{0}\right)>0 \text { and } u_{t}\left(x_{c}^{+}, t_{0}\right)<0
$$

The maximum principle in the paper region gives that $f\left(x_{c}, t_{0}\right)$ is also minimal with respect to $Q_{t^{*}}^{p}$, giving

$$
f_{x}\left(x_{c}^{-}, t_{0}\right)<0 \text { and } u_{t}\left(x_{c}^{-}, t_{0}\right)>0
$$

The second inequality in (6.4) and (6.5) contradict pressure continuity across the paper-felt contact. Hence $f>0$ in $Q_{t^{*}}^{f}$ yielding (6.3).

Next we show that paper loses water in the press-section and cannot be very thin unless the total pressure becomes large.

Proposition 6.2 For any $t>0$ we have

$$
1-\underline{\epsilon}<\frac{h_{p}(t)}{h_{p}(0)}<1
$$

where $\underline{\epsilon}=u_{0 p}-\underline{u}$ with $\underline{u}$ given in (4.17).

Proof. First note that $h_{p}(0)=x_{c}\left(u_{0 p}+1-\phi_{0 p}\right)=x_{c}$. Further, inequality (4.25) and the maximum principle give

$$
\underline{u}<u<u_{0 p} \text { in } Q^{p}
$$

Applying these bounds in (6.1) gives the desired estimates. 


\section{Numerical examples}

In this section we show the results of two numerical experiments. For the structural pressure we take, see also [15],

$$
P_{i}(u)=P_{i}^{*}\left(u^{-r_{i}}-u_{0}^{-r_{i}}\right), i=p, f
$$

The permeability is modelled by the Kozeny-Carman relation, see for instance [1],

$$
k_{i}(\phi)=k_{0 i} \frac{\phi^{3}}{(1-\phi)^{2}}, i=p, f .
$$

Here $P_{i}^{*}, r_{i}$ and $k_{0 i}$ are positive constants depending on the medium.

The experiments share the following values for the physical constants:

$$
\begin{aligned}
& h_{0 p}=2 \cdot 10^{-4} \mathrm{~m}, h_{0 f}=1.2 \cdot 10^{-3} \mathrm{~m}, t_{\text {fin }}=5.4 \cdot 10^{-2} \mathrm{~s}, u_{0 p}=0.55, u_{0 f}=0.42, P_{p}^{*}=1 \cdot 10^{5} \mathrm{~Pa}, \\
& P_{f}^{*}=2.2 \cdot 10^{5} \mathrm{~Pa}, r_{p}=5.15, r_{f}=2.7, \mu=10^{-3} \mathrm{~kg} \mathrm{~m}^{-1} \mathrm{~s}^{-1}, k_{0 p}=4.3 \cdot 10^{-16} \mathrm{~m}^{2} .
\end{aligned}
$$

Further, the total applied pressure is given by

$$
P_{T}(t)=6 \cdot 10^{6} \sin ^{2}\left(\pi t / t_{f i n}\right) \text { Pa for } 0 \leq t \leq t_{f i n} .
$$

With this particular choice, we consider two types of felt:

1. $k_{0 f}=4.1 \cdot 10^{-15} \mathrm{~m}^{2}$,

2. $k_{0 f}=8.2 \cdot 10^{-15} \mathrm{~m}^{2}$.

We combine the forward Euler method with the finite volumes discretization to approximate $u$ in the interior of each subdomain. At the interface we consider two additional unknowns, $u^{+}$and $u^{-}$, which are determined at each time step by imposing pressure and flux continuity across the interface. This yields a system of two equations in $u^{+}$and $u^{-}$(see [5] for details).
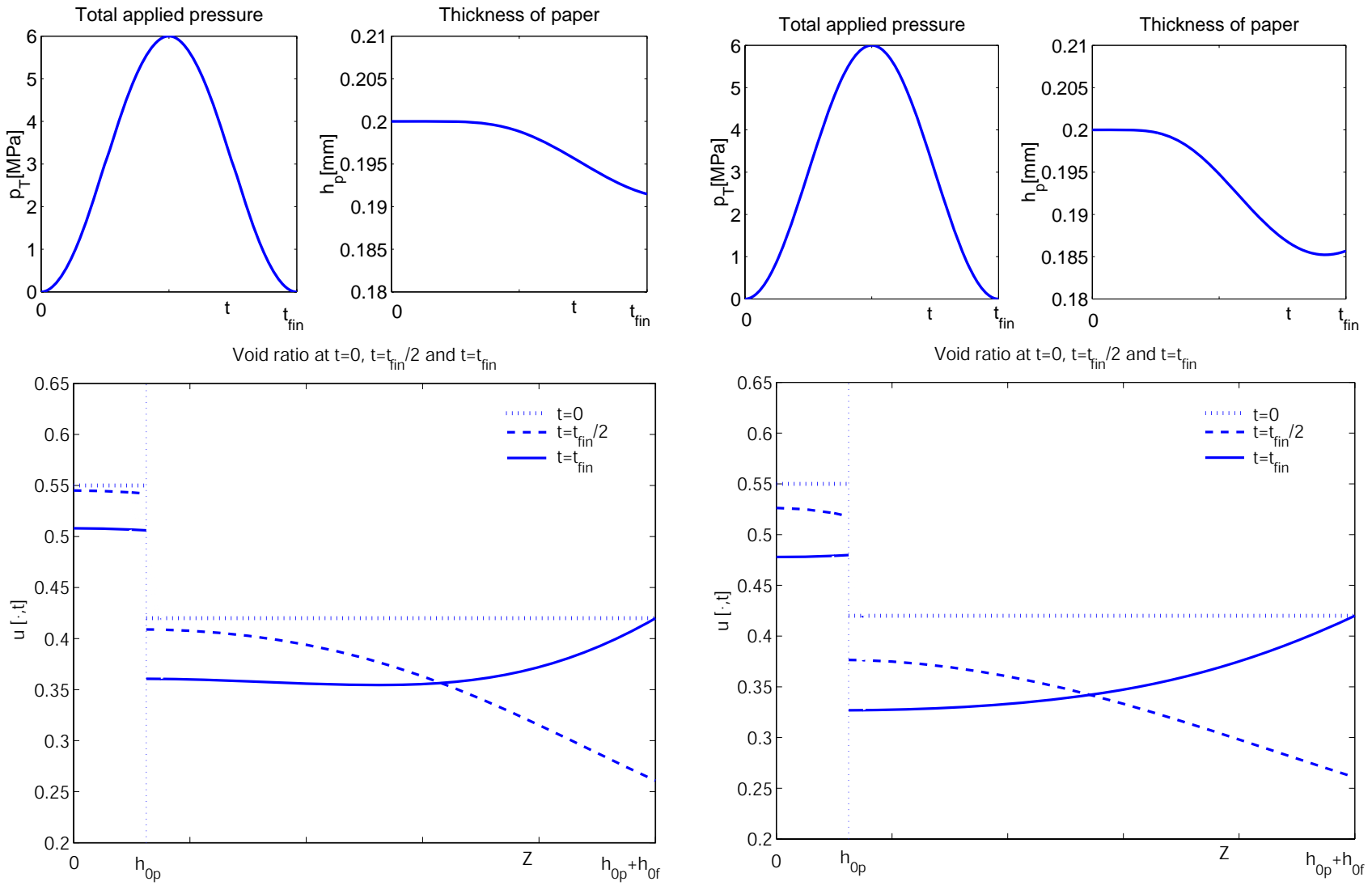

Fig. 6 Numerical results corresponding to case 1.

Fig. 7 Numerical results corresponding to case 2. 


\section{Comments:}

The first case corresponds to a relatively low felt permeability. The computational results are shown in Figure 6 . We observe that the paper thickness decreases during the passage through the press-section and that $u$ decreases from left to right. In other words, the water is flowing from paper to felt. This corresponds to identity (6.2).

The second case corresponds to a higher permeability. The computational results are shown in Figure 7 . Here we observe that paper expands before reaching the end of the press-section and that the water flow reverses direction. This phenomenon is called rewetting. In spite of this, the wet-pressing process is now more efficient than in the first experiment, since here $h_{p}(1)$ attains a lower value. The reason for this is that the higher felt permeability causes stronger diffusion and consequently a more efficient removal of water from the paper.

Acknowledgements This work was supported by the Netherlands Organization for Scientific Research (NWO), through the project 613.002 .046

\section{References}

[1] J. Bear, Dynamics of Fluids in Porous Media, Elsevier, New York (1972).

[2] M. Bertsch, R. dal Passo and C.J. van Duijn, Analysis of oil trapping in porous media flow, SIAM Journal on Mathematical Analysis, Volume 35 (1), 245-267 (2003).

[3] D. Bežanović, E.F. Kaaschieter and C.J. van Duijn, A three-phase model for wet pressing of paper, The Proceedings of 2003 International Paper Physics Conference, British Columbia (2003).

[4] C.J. van Duijn, Nonlinear Diffusion Problems, PhD thesis, University Leiden (1979).

[5] C.J. van Duijn, A. Mikelić and I.S. Pop, Effective equations for two-phase flow with trapping on the micro scale, SIAM J. Appl. Math., Volume 62 (5), 1531-1568 (2002).

[6] C.J. van Duijn and L.A. Peletier, Nonstationary filtration in partially saturated media, Archive Rat. Mech. Anal. 78, 173-198 (1982).

[7] A. Friedman, Partial Differential Equations of Parabolic Tipe, Prentice-Hall, Englewood Cliffs, New Jersey (1964).

[8] B.H. Gilding, Hölder continuity of solutions of parabolic equations, J. London Math. Soc. (2) 13, 103-106 (1976).

[9] R. Helmig, Multiphase Flow and Transport Processes in the Subsurface, Springer, Berlin (1997).

[10] K. Hiltunen, Mathematical Modelling for Consolidation Process in Paper Machines, PhD thesis, University of Jyväskylä, Finland (1995).

[11] F. Hirsch and G. Lecombe, Elements of Functional Analysis, Springer, New York (1999).

[12] M. Kataja, Hydrostatic and structural pressure in compressed paper webs and paper felts, Nordic Pulp and Paper Research Journal 3, $162-166$ (1995).

[13] M. Kataja, K. Hiltunen and J. Timonen, Flow of water and air in a compressible porous medium. A model for wet pressing of air, J. Phys. D: Appl. Phys. 25, 1053-1063 (1992).

[14] O.A. Ladyzhenskaya, V.A. Solonnikov and N.N. Uraltceva, Linear and Quasilinear Equations of Parabolic Type, Amer. Math. Soc. Transl. Math. Mono 23, Providence, R.I. (1968).

[15] B.M.P. Mulder and M. Riepen, Wet press modelling, a one-dimensional approach, Report to Netherlands Agency for Energy and Enivironment, project nr. 96.13.6.3201, Delft (1994).

[16] P. Nilson and K.O. Larson, Paper web performance in a press nip, Pulp and Paper Magazine of Canada, Deceber 20, 66-73 (1968).

[17] H. Paulapuro, Wet pressing-present understanding and future challenges, 12th Fundamental Research Symposium, Oxford (2001).

[18] M.H. Protter and H.F. Weinberger, Maximum Principles in Differential Equations, Prentice-Hall, Englewood Cliffs, New Jersey (1967).

[19] M. Riepen, An inside view on impulse drying phenomena by modelling, Report to TNO Institute of Applied Physics (1994).

[20] K.M. Singh, Mathematical Analysis of the Wet Pressing of Paper, PhD thesis, SUNY College of Environmental Science and Forestry, Syracuse, New York (1994).

[21] K. Velten and W. Best, Rolling of unsaturated porous materials: Evolution of fully saturated zone, Physical Review E, Volume 62 (3), 3891-3899 (2002). 